\title{
Burnout Syndrome in the Context of Entropy Neuron-Glial Networks of the Brain
}

\author{
Rosman $\mathrm{SV}^{1,2 *}$ \\ ${ }^{1}$ Physician of functional diagnostics of SBIH, Regional psychoneurological clinic, Russia \\ ${ }^{2}$ Head Psychiatric Department of SBIH, Regional psychoneurological clinic, Russia
}

Submission: August 04, 2017; Published: August 23, 2017

* Corresponding author: Rosman SV, Physician of functional diagnostics of SBIH, Regional psychoneurological clinic, Tver, Russian Federation Russia, Tel: +7-903-800-11-05; Email: seros2005@mail.ru

\begin{abstract}
The articles discusses the diagnosis of SPV and define its place among the other mental illnesses with new unique method of dispersion of amplitude-frequency characteristics of the alpha rhythm. The technique allows moving from categorical methods of diagnosis in psychiatry to demensial, to a certain extent, providing the search of a new paradigm.

Keywords: Professional burnout syndrome; Dispersion of alpha-rhythm; Diagnosis of mental illness

Abbreviations: BPD: Borderline personality disorder;BS:Burnout syndrome;DAFCAR: Dispersion of Amplitude-Frequency Characteristics of the alpha rhythmEEG; NGNB: Neuron-Glial Network of the brain; HVT: Hyperventilation test; CD $\alpha$ l: Coefficient of Dispersion of alpha-Rhythm EEG-1(the quotient of the modal values of power of alpha rhythm to his total power in the range of 7-13 Hz); CD 2: Coefficient of Dispersion of the alpha-Rhythm EEG-2 (the quotient of the power of the alpha rhythm in the range of "a modal value $\pm 0.5 \mathrm{~Hz}$ " to his total power in the range of 7-13 Hz);0 Mo f: Value of the Modal Frequencies in OccipitalElectrodes; F Mo f: Value of the Modal Frequencies in Frontal Electrodes; 0 Mo $\mathrm{f}$ - F Mo f : Value of the Difference of Modal Frequencies Between the Occipital and Frontal Electrodes; IIDA: Integral Index of Dispersionof the Alpha rhythmEEG(Value of the Kurtosis of the Normal Distribution CD $\alpha$ in the Occipital Electrodes); ADA: AsymmetryDistributionof the Alpha rhythmEEG (Value of the symmetryDistribution CD $\alpha$ in the Occipital Electrodes); IIH: Value of the Index Hypofrontality (Kurtosis of the Normal Distribution CD $\alpha$ in the Frontal Electrodes); AH: Value of the Asymmetry of CD $\alpha$ lin the Frontal Electrodes; CV\% - the Coefficient of Variation; CI: Confidence Interval; c.u.: Conditional Unit
\end{abstract}

\section{Introduction}

The problem of "burnout" in social terms goes far beyond the production problems. The world Health Organization considers the disease a "global epidemic", estimating the annual loss from her 200.000.000.000\$. Because we are all participants in the proceedings, and sick pay, hospitalization, and costs from reduced productivity are heavy burdens on the state budget. Social consequences of "burnout" of the President in historical terms can cost the lives of millions of its inhabitants and the inhabitants of neighboring countries, and change the historical fate of continents. And this is no exaggeration $[1,2]$.

In history, led many States were often personality, which fully fit the criteria of "burnout", or simply a mental disorder. However, we have no data on the survey of presidents and kings, so in the continuation of cycle of articles, devoted to a new method of studying the entropy of the NHS GM, we race-look, what are the signs of DAFCAR is characterized by the syndrome of professional burnout among people of ordinary professions, interpolating the data obtained at all working professionally in various fields of people. Burnout syndrome (BS) was first described in 1974, the American psycho log Freudenberger [3] to describe the demoralization, frustration and extreme fatigue, which he watched from mental health workers. The developed model proved to be convenient to evaluate this condition among health care workers occupations with the highest tendency to "burnout" because their working day is a constant close contact with people, besides the pain-governmental, requiring constant care and attention, restraint. The main symptoms of BS are:

a. weariness, fatigue, exhaustion after an active professional activity;

b. psychosomatic problems (fluctuations in blood pressure, headaches, digestive and cardiovascular systems, neurological disorders, insomnia);

c. emergence of a negative attitude to colleagues and clients (instead of the existing positive relationship) 
d. negative attitude to the activity performed;

e. aggressive tendencies (anger and irritability towards coworkers and clients);

f. functional, negative attitude;

g. anxiety, pessimistic mood, depression, sense of meaninglessness of the events, the guilt [1-14].

I think it does not make sense to describe in detail the clinical characteristics of the burnout syndrome. It is widely represented in the scientific and popular literature. Very ha Acterna formation in patients with a specific behavioral pattern:

a) the high tempo of life to achieve ill-defined (often elusive) goal;

b) constant pursuit of comparing and competition, to the promotion;

c) a persistent search of recognition;

d) active participation in different activities;

e) lack of time;

f) high commitment to action;

g) impatience and constant rush;

h) the sense of insecurity;

i) the high tempo of life and accelerate many physical and mental functions;

j) inability to relax.

Symptoms of this type of behavior: pointless hostility, irritability, rapid, loud, staccato speech, emphasizing certain words, expressions, energetic gestures and such people quickly go and quickly eat [7].

It should be noted that the overwhelming number of works devoted to professional burnout based on descriptive psychiatric and psychological techniques. Of course, there are also quite extensive neurophysiological researches on this issue. However, the disadvantage is of minor clinical value, because changes detected by these authors non-specific and rarely used as markers of psychopathology [6]. BS currently has the status of a diagnosis in the list of ICD-10 Z73 and Z56 Problems related to the difficulties of their lives and the problems associated with work and unemployment.

i. $\quad$ Z73 Problems related to life-management difficulty

ii. Z73.0 Burn-out

iii. Z73.2 Lack of relaxation and leisure

iv. Z73.4 Inadequate social skills, not elsewhere classified

v. Z73.5 Social role conflict, not elsewhere classified vi. Z56 Problems related to employment and unemployment

vii. Z56.3 Stressful work schedule

viii. Z56.4 Discord with boss and workmates

ix. Z56.5 Uncongenial work

x. Z56.6 Other physical and mental strain related to work

xi. Z56.7 Other and unspecified problems related to employment

Mental burnout is understood as a professional crisis associated with the work as a whole and not only with interpersonal relationships in the process. Burnout can be equated with distress (anxiety, depression, hostility, anger) in the extreme and to the third stage about the future of the adaptation syndrome - the stage of exhaustion. Burnout is not just a result of stress a consequence of unmanaged stress [2]. In fact, diseases these are derived from the psychiatric articles in the section emotional and behavioral disorders. However, there is another point of view. As studies have shown, include the identified disorders to normal is not quite professionally, as surveyed, though continued to work, but they already had the signs of maladjustment in interpersonal and family conflicts. To account for this identified disorder cannot be attributed to normal.

They require correction, and in most cases, and the treatment, otherwise the person having the symptoms, become more marginalized, which can lead to care at a lower professional level, including refusal of work; there is a more profound violation of interpersonal relations (the destruction of families, quarrels with the children, breakup of a relationship with a significant inner circle and friends). Deeper manifestations of maladjustment, maintaining professional status, may be alcoholism, abuse of tranquilizers, it is possible to use professional access to medicines. Maladjustment can go up to suicidal tendencies and completed suicides. All this confirms the need to combine the identified symptoms in a separate nosological unit, which should be considered in the context of ICD-10 [7]. Thus, to establish the ownership of SPV to psychopathology is an important methodological problem of psychiatry, it is impossible without evidence of the role of organic and functional pathological changes in the brain in this disease.

a. The purpose of the study: to determine whether patients with BS signs of accelerated entropy NGNB.

\section{Materials and Methods}

Was studied 68 men and 64 women aged from 25 to 40 years who underwent inpatient psychiatric examination and comply with the clinical criteria of SPV. Their distribution by occupational category is presented in (Table 1). All of them performed EEG 


\section{Global Journal of Addiction \& Rehabilitation Medicine}

according to standard methods with the installation of the electrodes according to the international scheme "10-20\%" with ipsilateral reference electrodes with conducting HVT for 3 min with a frequency of $16 \mathrm{in}$-depth breaths in $1 \mathrm{~min}$. Clinical and

anamnestic data were collected on the basis of entries in the case histories. The obtained results were statistically processed using Microsoft Excel and Statistica 10.0 according to the method of determining the parameters DAFCAR [8].

Table 1: Distribution of patients according to professional categories.

\begin{tabular}{|c|c|c|c|c|c|c|}
\hline \multirow[t]{2}{*}{ Professional contingent } & \multicolumn{3}{|c|}{ Men, experience } & \multicolumn{3}{|c|}{ Women, experience } \\
\hline & 1-4 years & $5-9$ years & 10 years or more & 1-4 years & 5-9 years & 10 years or more \\
\hline Managers of different levels & & & 2 & & 2 & \\
\hline Health workers & & 2 & 5 & 1 & 5 & 10 \\
\hline University Professors & & 2 & 1 & & 3 & 3 \\
\hline Teachers of secondary schools & & 2 & 2 & 2 & 5 & 4 \\
\hline Educators of preschool institutions & & & & 4 & 6 & 12 \\
\hline Technical workers & 10 & 17 & 24 & & 3 & 2 \\
\hline Finance staff & & 1 & & 1 & 1 & \\
\hline TOTAL & 10 & 24 & 34 & 8 & 25 & 31 \\
\hline
\end{tabular}

Results

Analysis of the case histories revealed the following distribution of patients according to the criteria of BS (Tables 2-6).

\section{a. Discussion of the results}

Table 2: The distribution of the patients according to the criteria of BS.

\begin{tabular}{|c|c|c|}
\hline Criteriafor BS & Men & Women \\
\hline fatigue, fatigue, exhaustion after an active professional activities & 66 & 64 \\
\hline $\begin{array}{c}\text { psychosomatic problems (fluctuations in blood pressure, headaches, digestive and } \\
\text { cardiovascular systems, neurological disorders, insomnia) }\end{array}$ & 55 & 60 \\
\hline the emergence of negative attitudes to customers & 45 & 56 \\
\hline a negative attitude to the activity performed & 65 & 59 \\
\hline Aggressive tendencies (anger and irritability towards coworkers and customers) & 67 & 45 \\
\hline Auto aggression, negative attitude & 45 & 44 \\
\hline Anxiety, pessimistic mood, depression, sense of meaninglessness of the events, the guilt. & 64 & 62 \\
\hline
\end{tabular}

Table 3: The distribution of the patients a BS in clinical diagnostic syndromes.

\begin{tabular}{|c|c|c|}
\hline Clinical syndromes & Men & Women \\
\hline Asthenic and asthenic-hypochondriac & 51 & 48 \\
\hline reduction of professional duties and achievements & 62 & 10 \\
\hline neurotic depersonalization & 10 & 10 \\
\hline obsessive-phobic & 7 & 6 \\
\hline professional diluting or cynicism & 37 & 35 \\
\hline depressive different severity & & 22 \\
\hline of them : & 24 & \\
\hline disturbing option & 7 & 10 \\
\hline dysphoric & 27 \\
\hline apathetic & & 26 \\
\hline mixed & 50 \\
\hline Alcohol abuse & 14 & 3 \\
\hline
\end{tabular}




\section{Global Journal of Addiction \& Rehabilitation Medicine}

A General analysis of the contingent of the surveyed shows that the SPV group represented young people, socially successful, with higher and specialized secondary education and work experience up to 10 years. In spite of numerous psychological and psychosomatic problems (Table 2, таб3) according to EEG studies we do not find those changes that are characteristic of BPD [12]. Cartograms not different from normal, however in conjunction with the data (Tables 4-6) there is a slight increase of the indices DAFCAR compared to the norm, particularly in frontal leads(КДа1 F, IIG) with some trends of alpha rhythm slowing(decrease in $\mathrm{O}$ Mo F). This fact is very interesting, because it reflects the overall tendency of these patients to the anxiety and depression that was noted in the works of L. B. Ivanov [5], describing the pattern of EEG as "alarming".

Table 4: The summary table of parameters DAFCAR with BS in comparison with the norm and BPD, he left hemisphere.

\begin{tabular}{|c|c|c|c|c|c|c|c|}
\hline \multirow[b]{2}{*}{ Centhr } & \multirow[b]{2}{*}{ Indes DLFCA } & \multicolumn{2}{|l|}{4} & \multicolumn{2}{|l|}{ IIHD } & \multicolumn{2}{|c|}{ Marmu } \\
\hline & & 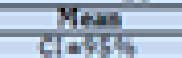 & 대개 & 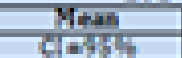 & 대로 & 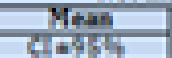 & CVT \\
\hline \multirow{16}{*}{$\mathrm{Mr}$} & Aphun/Ayt & $21-24$ & 37 & 1974:515 & 57 & intinis & 15 \\
\hline & Apband/Hpt & $\frac{1453}{1244}$ & $\$ 1$ & 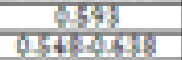 & 35 & prith & 19 \\
\hline & Aphas/Ayt & $018 \div$ & 120 & p112417 & 81 & 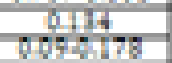 & 128 \\
\hline & FHat & 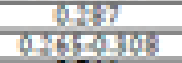 & 11 & 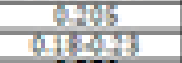 & 49 & phyt & 14 \\
\hline & Q Mof & Dinti & 9 & $\begin{array}{c}5373 \\
0458418\end{array}$ & 31 & $\frac{0745}{\text { prindis: }}$ & 9 \\
\hline & aMton & 617.9.9.5 & 37 & 2431517 & 111 & 6.845 & 10 \\
\hline & CGat & $\begin{array}{c}245 \\
252515\end{array}$ & 16 & 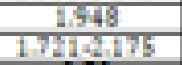 & 47 & $\frac{2 \pi 5}{15 n^{2}}$ & 12 \\
\hline & $\cos$ & $\begin{array}{l}18 \pi 5 \\
447-1034\end{array}$ & 6 & $\begin{array}{c}2.79 \\
458-106\end{array}$ & 9 & $\frac{1025}{1011-104}$ & 6 \\
\hline & $\cos 1 . F$ & 1SHy & $t$ & $\frac{342}{1453}$ & 12 & $\frac{1624}{1012-1041}$ & 5 \\
\hline & CEnt_F & bis: & 140 & DHAS & 217 & bint & 214 \\
\hline & AF $F$ & 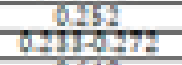 & 24 & Dinsting & 51 & 6123 & 25 \\
\hline & IItA & bets & 14 & $\begin{array}{c}\text { MA44 } \\
\text { D.114AB }\end{array}$ & $\$ 4$ & DEA & 14 \\
\hline & ABA & 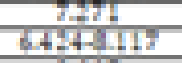 & \$1 & 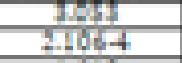 & $1 \pm 4$ & 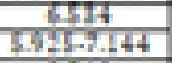 & 16 \\
\hline & 田 & IIAPII & 16 & This & $\$ 1$ & $\frac{242}{1+29}$ & 14 \\
\hline & $\mathbf{I}$ & क्या & 97 & b.44 & 109 & dift & 72 \\
\hline & Apt & 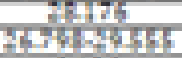 & 14 & AhH4 & 14 & 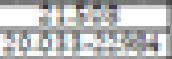 & 27 \\
\hline \multirow{16}{*}{ Worta } & Apha:HAph & bings & 50 & bit2d & 57 & blat & 40 \\
\hline & Aphs-7/Apl & artify & 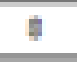 & 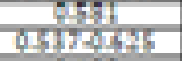 & $\$ 7$ & dats & 7 \\
\hline & Apha-1/Apl & ponting & 91 & Matish & 81 & atist & $6+$ \\
\hline & FHot & कin & 21 & brist & 4 & कमHA & 17 \\
\hline & QMof & brify & 8 & 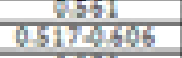 & 91 & prist & 9 \\
\hline & aMs[07] & 6589.917 & 40 & 28745 & 111 & 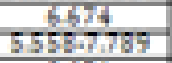 & 29 \\
\hline & CDal & 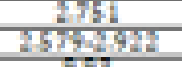 & 17 & 1504 & 47 & $\frac{25}{1+21}$ & 12 \\
\hline & $\cos$ & 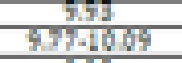 & 4 & $\begin{array}{c}757 \\
43748 \\
\end{array}$ & 9 & $\frac{1013}{48+1038}$ & 4 \\
\hline & CDa I_F & $\begin{array}{c}47 \\
4 \text { yndogh }\end{array}$ & 4 & 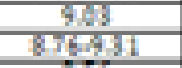 & 12 & 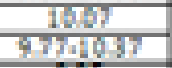 & 5 \\
\hline & CDEA_F & bothis & 140 & d55 & 217 & 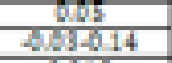 & 270 \\
\hline & AF.F & bess & 21 & 01315111 & 51 & 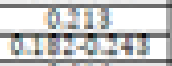 & 25 \\
\hline & ITA & 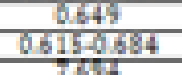 & 11 & DA44 & 14 & 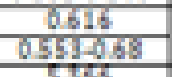 & 45 \\
\hline & ADA & 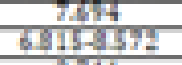 & 92 & 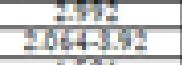 & 1.4 & $\begin{array}{c}\text { HMH } \\
4301+544\end{array}$ & 28 \\
\hline & 표 & IIIIII & 14 & 1.51 & 4 & 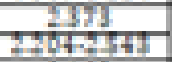 & 12 \\
\hline & $\mathbf{I}$ & 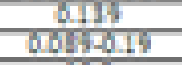 & 100 & DHAS & 105 & 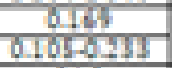 & 4 \\
\hline & At & $\frac{235}{4 P B C}$ & 17 & $\begin{array}{l}\text { M4P } \\
\text { MPSP }\end{array}$ & 14 & $\begin{array}{l}74 \\
414\end{array}$ & $\mathrm{M}$ \\
\hline
\end{tabular}


Global Journal of Addiction \& Rehabilitation Medicine

Table 5: The summary table of parameters DAFCAR with BS in comparison with the norm and BPD,the right hemisphere.

\begin{tabular}{|c|c|c|c|c|c|c|c|}
\hline \multirow{3}{*}{ Gridt } & \multirow{3}{*}{ IndenDAFCAR } & \multicolumn{2}{|l|}{ IIS } & \multicolumn{2}{|l|}{ IIHD } & \multicolumn{2}{|c|}{ Morm } \\
\hline & & N+⿱一𫝀口十 & \multirow{2}{*}{ an } & MFI & \multirow{2}{*}{ chis } & Mrin & \multirow{2}{*}{ a } \\
\hline & & Cat54 & & 대내 & & 대새 & \\
\hline \multirow{30}{*}{ Мн } & \multirow{2}{*}{ Aph-1/Aph } & 0114 & 45 & 194 & 63 & with & 18 \\
\hline & & WHAI & & onding & Wa & DADAMIII & 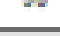 \\
\hline & 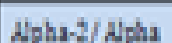 & 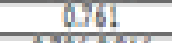 & 21 & 065 & t1 & MN & \\
\hline & & DHESA & in & DMAGL & $n$ & DMASI & A \\
\hline & diph $t /$ doth & dol & 14 & DIII & & DN & Hit \\
\hline & Арнтолар & THAB & $m$ & 4hanth & Yit & Pothents & m \\
\hline & Fust & tND & 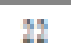 & HII & 44 & mi & 16 \\
\hline & & phath & Ma & HWHAN & 48 & PHAOH & is \\
\hline & $0 \mathrm{MaI}$ & 0731 & 11 & DSW & 94 & 275 & 4 \\
\hline & & bars & 12 & dy+464 & 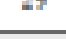 & b4ams & 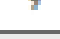 \\
\hline & 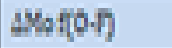 & 0401 & 40 & 46 & 14 & 1,117 & 11 \\
\hline & & IHI & & It5 & & $m_{4}$ & \\
\hline & $\cos$ & Whinht & if & WNHIII & W & 25124 & 13 \\
\hline & & 1006 & & 97 & & 1615 & \\
\hline & $\cos$ & GEISA & 7 & 4A4494 & $y$ & 1R1-10M4 & 5 \\
\hline & cosit & 445 & 7 & $42 H$ & ii & Math & 1 \\
\hline & $\cos \theta$ & 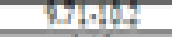 & 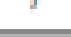 & पानH & H & 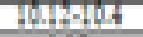 & \\
\hline & con F & 42 & 191 & 4 & 267 & 64 & 5 \\
\hline & tong & morst & ini & and & $=0$ & 6 mon & $\omega$ \\
\hline & Af & wh & 21 & dis & 5 & H & 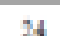 \\
\hline & 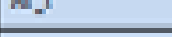 & 0131245 & in & 402415 & it & 615-1244 & 17 \\
\hline & BA & 0,045 & & 044 & & 15 & \\
\hline & $\omega n$ & 6indsh & $\omega$ & WHILA4B & 94 & WhसमS5 & $\pi$ \\
\hline & And & PIN & 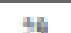 & 211 & $13^{4}$ & 114 & \\
\hline & $\operatorname{sen}$ & 440.15 & +9 & Mnsment & 19. & MHसम & 4 \\
\hline & 패 & 44 & $M$ & H4 & 58 & 247 & 7 \\
\hline & $=$ & 256128 & & 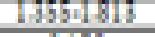 & & 271201 & $=$ \\
\hline & II. & 0115 & E8. & o1N & 74 & But & II \\
\hline & 4 & 617 눈 & 3 & Dheshit & in & WHEMU64 & M \\
\hline & $4 p$ & 2817 & 14 & $\frac{2517}{467+4 y}$ & 14 & 215H & 27 \\
\hline \multirow{30}{*}{ Whantan } & \multirow{2}{*}{ Apha-1/Apu } & DII4 & \multirow[b]{2}{*}{$n$} & WH & \multirow[b]{2}{*}{61} & SII & \\
\hline & & Whesins & & WHIMH & & DWHA.44 & $\$$ \\
\hline & 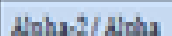 & ants & 0 & 154 & 11 & int & 8 \\
\hline & 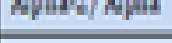 & 6rHSTH & $y$ & 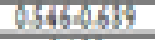 & M & whs & $y^{2}$ \\
\hline & Aph-S/Aph & 046 & 64 & 9164 & 92 & rest & 5 \\
\hline & Арнат Арн & 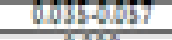 & 34 & 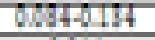 & 7 & 604J-1617? & $\mathrm{M}$ \\
\hline & fust & 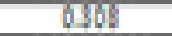 & \#1 & 1211 & At & $\Delta M$ & 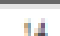 \\
\hline & Fat & 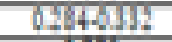 & it & DNADA14 & tis & DMADMA & 24 \\
\hline & byot & Tht & HI & Hit & 3 & Whit & A \\
\hline & qhat & when & $\pi$ & WHESH & in & whollt & 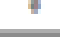 \\
\hline & Мatof) & Hat & 35 & shy & 86 & 401 & 24 \\
\hline & & Mathen & & Mation & & 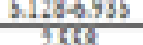 & \\
\hline & Ea! & $25 B M B M$ & 11 & 154217 & 40 & & 11 \\
\hline & & PII & & it & & Thir & \\
\hline & cent & Mhem & $\Rightarrow$ & IHW & 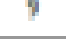 & FHoth & t \\
\hline & ODy & W & 5 & in & 11 & DW & f \\
\hline & whang & $44,4 \%$ & 2 & Hot & $\pi$ & when & 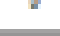 \\
\hline & CDEF & 008 & 151 & $\frac{017}{104}$ & 267 & 67 & 25 \\
\hline & & 0121 & & Dist & & मnS & \\
\hline & Al & प195m & $\pi$ & WHAMT & 32 & 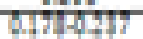 & 24 \\
\hline & DA & W4 & 15 & 14 & 34 & MI & I8 \\
\hline & IA & WHEWH & ab & dfupdit & st & DARATh & $\mathrm{m}$ \\
\hline & ADA & 755 & 11 & 2842 & & 457 & \\
\hline & BDA & BSAS45 & M & 19BमS45 & IMi & DHSH & 11: \\
\hline & 国 & 1714 & 15 & 155 & 58 & HEI & 45 \\
\hline & & Hatin & & 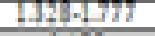 & & $2015-47$ & \\
\hline & I & bu & 67 & DH & 70 & $\Delta \mu$ & $B$ \\
\hline & & 147454 & 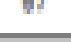 & W.14DWh & $m$ & DIA4 & \\
\hline & Ay & 2111 & 11 & AI & it & An & 15 \\
\hline & $4^{2}$ & WEH & & When & & 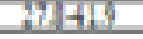 & \\
\hline
\end{tabular}




\section{Global Journal of Addiction \& Rehabilitation Medicine}

Table 6: Comparative indicators of differences in the average values of certain parameters of the BS compared with normal by Student's test (t-value).

\begin{tabular}{|l|c|c|r|r|r|r|r|}
\hline \multicolumn{1}{|c|}{ Index DAFCAR } & \multirow{2}{*}{ Gender } & \multirow{2}{*}{ Hemisphere } & Mean & Mean & \multirow{2}{*}{ twalue } & \multirow{2}{*}{ df } & \multirow{2}{*}{ p } \\
& & BS & NORMA & & \\
\hline CDa1 F & men & left & 0.25233 & 0.22963 & 1.99424 & 93 & 0.049054 \\
\hline O Mof - F Mof & men & left & 0.10985 & 0.04098 & 2.60665 & 93 & 0.010649 \\
\hline CDa2 & men & right & 0.73312 & 0.76716 & -2.01705 & 92 & 0.046605 \\
\hline IIH & men & right & 7.31903 & 6.19448 & 2.13670 & 92 & 0.035276 \\
\hline AH & men & right & 2.68438 & 2.47891 & 2.08738 & 92 & 0.039619 \\
\hline F Mof & men & right & 9.95409 & 10.26250 & -2.37560 & 92 & 0.019595 \\
\hline O Mof - F Mof & men & right & 0.11699 & 0.05000 & 2.55395 & 92 & 0.012295 \\
\hline CDa1 F & women & left & 0.25795 & 0.21252 & 2.64318 & 44 & 0.011336 \\
\hline IIH & women & left & 7.69359 & 5.36648 & 3.30394 & 44 & 0.001901 \\
\hline AH & women & left & 2.74079 & 2.37313 & 3.10866 & 44 & 0.003290 \\
\hline CDa1 F & women & right & 0.25720 & 0.20755 & 2.72663 & 44 & 0.009153 \\
\hline IIH & women & right & 7.55302 & 4.99323 & 3.33681 & 44 & 0.001730 \\
\hline AH & women & right & 2.71374 & 2.28147 & 3.14723 & 44 & 0.002956 \\
\hline O Mof & women & right & 9.84636 & 10.16071 & -2.01666 & 44 & 0.049860 \\
\hline
\end{tabular}

Note. Values highlighted in red font are statistically significant differences in the compared groups.
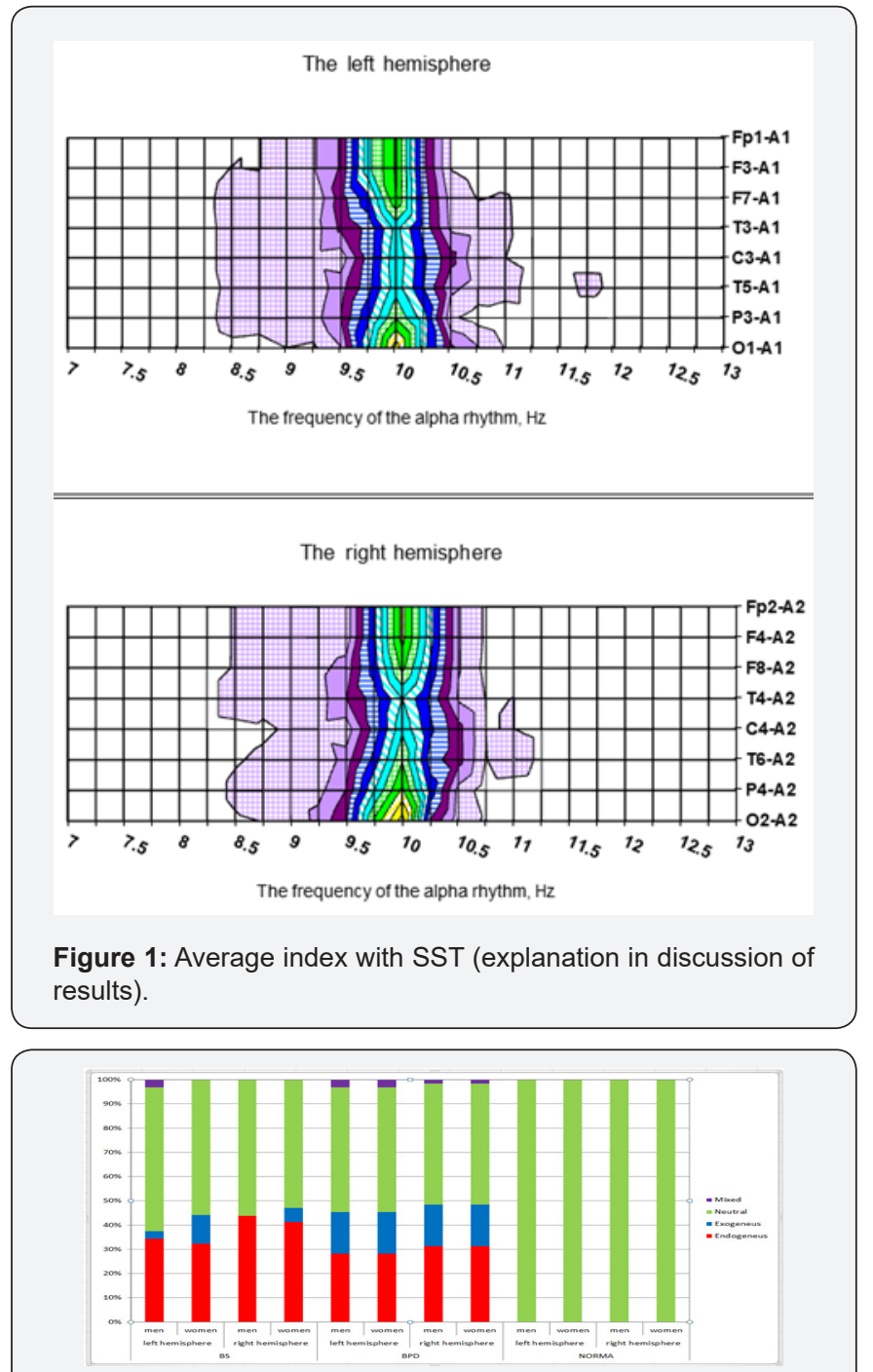

Figure 2: The structural distribution of the results of HVT while BS and BPD according to the types of reaction.
In all likelihood, this process reflects a compensatory reaction to a stressful mechanisms, which are aimed at preservation of stability and synchronization of work of all Department of the brain. Such "functional supersynchronous" brain activity for some time is able to maintain stability and efficiency. However, resources such mode of operation is very limited (Figure 1). Accordingly, all parameters DAFCAR when BS is much higher than those with BPD, thus it is doubtful that BS belonged to the BPD group. An interesting situation arises - BS when we have a normal EEG with elements of "a disturbing pattern". However, the results of the assessment data show HVT, that we are dealing with subcompensated disorders, and this is confirmed by the analysis of structural distribution of the results of HVT for the types of reaction. It is clearly seen that these results with BS are almost identical to the results in BPD (Figure 2). There is even a structural predominance of the endogenous reaction in BS, but most likely, if the BPD has a prevalence of exogenous pathology of vascular and toxic Genesis in patients of this category, a very high percentage of alcoholism and asocial. This may be caused by the following reasons:

a. Changes to the NGNB at subcompensated BS are subclinical in nature

b. There is a constitutional defect NGNB, which leads to destabilizing go-the criminal brain.

Previous articles in this series $[8,9,12,13]$ on DAFCAR emphasized repeatedly on the stability of endogenous reactions in ontogenesis. Of course, this is only a hypothesis, but it is possible that here we are faced with the true cause of certain mental illnesses. No one doubts that schizophrenia is transmitted genetically, however, the gene for schizophrenia has never been found. Perhaps, genetically transmitted functional failure of the frontal lobes to respond adequately to stress - instead of the synchronized activities of neurons occurs disrupting their work. 
Thus, dopamine synchronization mechanism of the brain leads to the opposite effect.

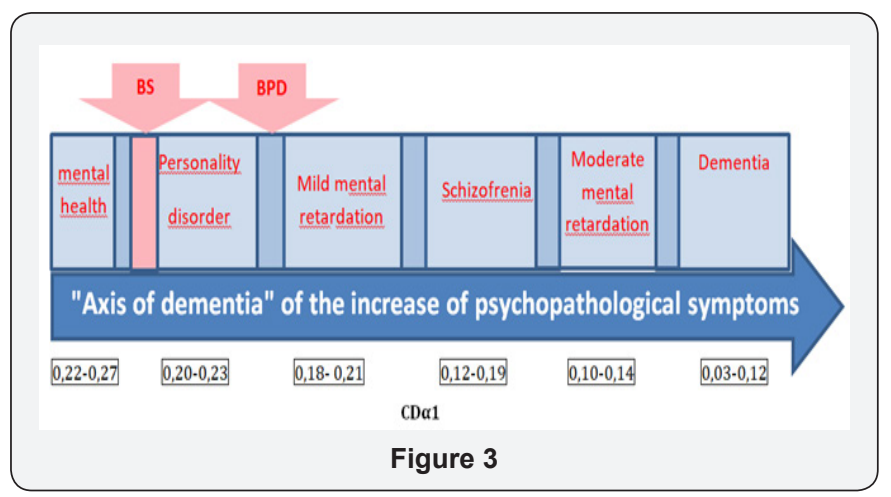

When a BS picture of imaginary EEG of well-being in which the entropy NGNB are in the intermediate stage, subclinical, so the "axis of dementia" BS is directly next to the norm, but the norm is no longer (Figure 3). Independent confirmation of these results could have very significant implications for the evaluation of patients to identify potential candidates for the SPV group. This has implications for the prediction of stress examined for inclusion in the composition of the contingent employed in the heavy and responsible operations. Preventive examination in the clinical examination will allow you to diagnose hidden psychopathology that will help to reduce the incidence of such socially significant diseases as depression, drug addiction and alcoholism, will reduce the level of suicides.

Currently, the possibility of this is greatly facilitated by using a new instrument, which is based on the identification of the degree of entropy NGNB according to the method of determining indices of DAFCAR- "Detector of the neuropsychiatric disorders" produced by MCS (Zelenograd, Russia). Of course, the question may arise why the dispersion characteristics of the alpha rhythm are not absolute? The answer is very simple: first, despite the fact that in a series of articles dedicated to the new method of the study has been proved repeatedly the ability of the indexes DAFCAR to identify psychopathology, it cannot be assumed that this token entropy NGNB - only; second, those who read an article on fractal mechanism of the brain[8], it should be understood that using the classic EEG we see not three-dimensional and two-dimensional distribution pattern DAFCAR, and therefore much of the information is hidden from us, because the neurophysiological fractal of the brain is three-dimensional [8].

We still have a lot should learn - for example, to link DAFCAR with the phase changes of the oscillations of the potentials(a measure of coherence) in the brain, to understand the mechanisms of regulation of the number of iterations in NGNBbefore we can talk about the complex markers of entropy NGNB. But the most important thing is to ensure that potentials in the whole volume of the brain non-invasive way.

\section{Conclusion}

a. Patients with BS are primary, sub compensated, signs of entropy NGNB, which can be verified using EEG HVT according to S. V. Rosman (2017).

b. It is not excluded that BS patients before any clinical symptoms had a predisposition to this disease in the form of hidden functional inferiority of the NGNB, which may be uncompensated as a result of distress.

c. Early diagnosis of the initial stages of the BS by using a new method of identifying entropy NGNB - dispersion of amplitude-frequency characteristics of the alpha rhythm, will help to timely identify patients with the goal of preventing, in the process of treatment, the severe social consequences of the BS -suicide, crime, alcoholism, drug abuse and significant economic losses.

\section{References}

1. De Mercato R, Celentano, A Romano, D Guarnaccia, M Di Pietro, et al. (1995) Burnout syndrome in medical and non medical staff . New Trend in Experimental and Clinical Psychiatry 11(1): 43-45.

2. Dietzel, Laurie C, Coursey, Robert D (1998) Predictors of emotional exhaustion among nonresidential staff persons 21(4): 340-348.

3. Freudenberger (1974) HJ Staff burnout. Journal of Social Issues 30: 159-165.

4. Grabe M (2008) burnout Syndrome the disease of our time, M Grabe: per s nem, edited by LG Lysyuk - SPb: Speech pp. 96

5. Ivanov LB (2016) Psyco-phisiological interpretation of the functional state of the brain using EEG. Bullet Of clin Neurophysiolog (1): 5-26.

6. Kondratyeva OG (2010) Changes of alpha-rhythm EEG in the formation of of burnout syndrome of teachers of secondary schools. Bulletin of the Chelyabinsk state pedagogical University 9: 263-270

7. (2013) Professional burnout syndrome: prevention and correction. Methodical manual. Kaluga: Scientific-methodical and educational innovative center Health.

8. Rosman S (2017) The Theoretical Foundations of Dispersion of Amplitude-Frequency Characteristics of the Alpha Rhythm of the EEG. Glob J Add \& Rehab Med 2(3).

9. Rosman S (2017) The Use of Analysis of Variance of the Alpha Rhythm of the EEG in the Study of the Pathogenesis of Alcoholism and the Causes of Alcoholic Deliria. Glob J Add \& Rehab Med 2(1): 555580.

10. Rosman SV (2013) Diagnostic capabilities of dispersion mapping the alpha rhythm of the electroencephalogram. Mental health 6: 64-69.

11. Rosman SV (2013) Opportunities of the dispersive mapping of the alpha rhythm of electroencephalogram in diagnostics of schizophrenia. Psychiatry 2: 32-37.

12. Rosman SV (2017) Borderline Personality Disorder in the Context of Entropy Neuron-Glial Networks of the Brain. Glob J Add \& Rehab Med 2(4): 555595

13. Rosman S, Kurakhina O (2017) Violent Crime in the Context of Entropy Neuron-Glial Networks of the Brain. Glob J Add \& Rehab Med 2(5): 555599 .

14. Sazonov VY (2012) Burnout in oncologists, features of its formation and prevention. 
This work is licensed under Creative Commons Attribution 4.0 License

DOI: 10.19080/GJARM.2017.03.555611
Your next submission with Juniper Publishers will reach you the below assets

- Quality Editorial service

- Swift Peer Review

- Reprints availability

- E-prints Service

- Manuscript Podcast for convenient understanding

- Global attainment for your research

- Manuscript accessibility in different formats ( Pdf, E-pub, Full Text, Audio)

- Unceasing customer service

Track the below URL for one-step submission https://juniperpublishers.com/online-submission.php 